\title{
Single-Nucleotide Polymorphism Detection Based on a Temperature-Controllable Electrowetting on Dielectrics Digital Microfluidic System
}

\author{
Hsien-Hua Shen ${ }^{1}$, Tsung-Yao Su${ }^{1}$, Yi-Ju Liu², \\ Hwan-You Chang ${ }^{3}$ and Da-Jeng Yao ${ }^{1,4, *}$ \\ ${ }^{1}$ Institute of NanoEngineering and MicroSystems, National Tsing Hua University, \\ Hsinchu 30013, Taiwan, R.O.C. \\ ${ }^{2}$ Food Industry Research and Development Institute, \\ Hsinchu 30062, Taiwan, R.O.C. \\ ${ }^{3}$ Institute of Molecular Medicine, National Tsing Hua University, \\ Hsinchu 30013, Taiwan, R.O.C. \\ ${ }^{4}$ Department of Power Mechanical Engineering, National Tsing Hua University, \\ Hsinchu, Taiwan, R.O.C.
}

(Received May 30, 2013; accepted August 1, 2013)

Key words: electrowetting on dielectric, microfluidics for biomedical applications, microheater, single-nucleotide polymorphism, lab-on-chip

To use efficiently a digital microfluidic system with an electrowetting on a dielectric (EWOD) technique to detect a single-nucleotide polymorphism (SNP) in a specific gene sequence, we designed microheaters on the cover of an EWOD chip for temperature control of a bioreaction. The droplets on an EWOD chip were actuated with addressable electrodes. Multiple bioreagents such as DNA, micromagnetic beads, and buffer droplets have been manipulated using the EWOD system. Magnetic beads (MBs), whose magnetic property enabled them to be collected and purified with an applied magnetic field, were used to detect an SNP in a specific gene sequence. The microheaters were fabricated on a glass substrate with Pt deposition. After designing the heater circuit, the top plate was constructed with a bottom chip to complete the packaging of a temperature-controllable EWOD system. These microheaters in an EWOD system can provide an appropriate temperature for DNA ligase in experiments to detect an SNP. The temperature-controllable EWOD digital microfluidic system detected an SNP code in a specific gene sequence. This research will contribute to biomedical diagnosis through a lab-on-a-chip approach.

*Corresponding author: e-mail: djyao@mx.nthu.edu.tw 


\section{Introduction}

In recent years, micro- and nano-electromechanical systems (MEMS/NEMS) technologies have been applied to portable point-of-care (POCT) diagnostic devices. Chemical sensors, ${ }^{(1,2)}$ microfluidic channels, ${ }^{(3,4)}$ and neural microprobes ${ }^{(5,6)}$ are examples of diagnostic tools that have been extensively developed and aimed for rapid and accurate detection. Diagnosis based on a technique involving electrowetting on a dielectric (EWOD) has become important in a digital microfluidic system. EWOD has already proven its potential within a broad range of bioassays. ${ }^{(7-9)}$ An electric field can induce an electric double layer in aqueous droplets, which can decrease the contact angle between droplets and the hydrophobic surface, changing a hydrophobic area into a hydrophilic area; this phenomenon is called electrowetting. The relationship between applied voltages and contact angle changes can be determined by combining Lippmann's equation and Young's equation, shown in eq. (1). Here, $\theta(\mathrm{V})$ and $\theta^{\circ}$ are the corresponding contact angles at applied voltages $V$ and $0 \mathrm{~V}, \varepsilon_{\mathrm{r}}$ and $\varepsilon_{0}$ are the dielectric constants of the insulating layer and the vacuum permittivity, respectively, $\gamma_{\mathrm{LG}}$ is the liquid-gas interfacial energy, and $t$ is the thickness of the dielectric layer. ${ }^{(10)}$

$$
\cos \theta(\mathrm{V})-\cos \theta^{\circ}=\frac{\varepsilon_{\mathrm{r}} \varepsilon_{0}}{2 \gamma_{\mathrm{LG}} t} V^{2}
$$

When electrodes are patterned, unit-sized packets of fluid can be created, transported, merged, and cut by changing the wettability of droplets on a hydrophobic surface. ${ }^{(11)}$ The advantages of the EWOD digital microfluidic system are simple fabrication, low power consumption, and convenient manipulation of droplets. Many studies on the biomedical application of an EWOD system have been published. ${ }^{(12)}$ Liu et al. and Lin et al. accomplished DNA ligation ${ }^{(13,14)}$ and magnetic bead-based DNA detection ${ }^{(15)}$ on an EWOD microfluidic system; the volume of a bioreagent was greatly decreased through EWOD droplet manipulation. Shah's research group used antibody-modified magnetic beads to recognize CD8 ${ }^{+}$T-lymphocytes on EWOD chips. ${ }^{(16)}$ Moreover, EWOD is also used in point-of-care diagnosis such as chemical extraction, ${ }^{(17)}$ protein crystallization, ${ }^{(18)}$ immunoassays, ${ }^{(19,20)}$ and cell-based assays. ${ }^{(21)}$

In these applications, some require a suitable reaction temperature. A water bath or oven is typically used as an additional source of heat, but some scientists tried to design heating devices within their EWOD system to replace traditional sources of heat. For example, Nelson and coworkers designed locally heated EWOD electrodes for temperature control.(18) The EWOD chip provided a suitable reaction temperature for trypsin digestion, sample drying, and matrix crystallization. In this paper, we designed MEMS Pt resistive microheaters on the cover of an EWOD chip. ${ }^{(22,23)}$ Figure 1(a) shows the concept of the temperature-controllable EWOD microfluidic device. The resistive microheaters have the advantages of low power consumption and a very short response time, which make a microfluidics system more flexible in various biomedical reactions. We achieved the detection of single-nucleotide polymorphism in an experiment to demonstrate the temperature-controllable EWOD systems. 


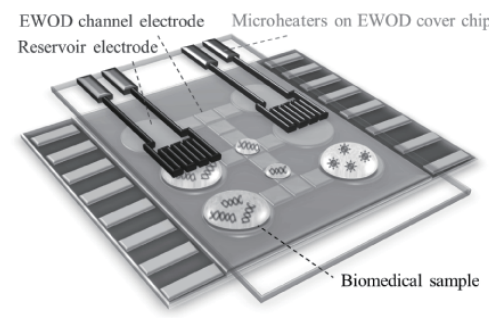

(a)

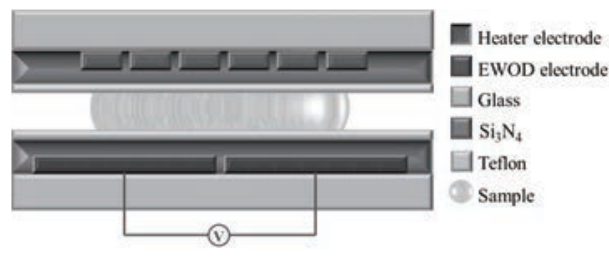

(b)

Fig. 1. Temperature-controllable EWOD digital microfluidic system. (a) Schematic diagram of EWOD device with microheaters. (b) Cross section of coplanar-type electrodes of EWOD chip with microheaters.

\section{Materials and Methods}

\subsection{EWOD system with coplanar electrodes}

An EWOD digital microfluidic channel with coplanar electrodes was used in this research. Two different types of electrode including reservoir and channel electrode, were designed for droplet manipulation. An advantage of coplanar electrodes in the EWOD digital microfluidic system is that there is no electrode pattern on the cover; another pattern such as a microheater can thereby be designed on that cover. The cross section of an EWOD chip with coplanar electrodes is shown in Fig. 1(b). After indium tin oxide (ITO) electrodes were patterned by photolithography, one layer of plasmaenhanced chemical-vapor-deposited (PECVD) $\mathrm{Si}_{3} \mathrm{~N}_{4}$ (450 nm thickness) and a spinon-glass (SOG) thin film (100 nm thickness) were spin coated on an ITO electrode as a compound dielectric. Finally, one layer of Teflon (150 nm thickness) was spin coated on the dielectric layer as a hydrophobic layer. Between the EWOD chip and the hydrophobic cap, one layer of $70 \mu \mathrm{m}$ double-sided tape was used as the spacer. Droplets were manipulated by four basic actuation methods: creation, transport, merging, and

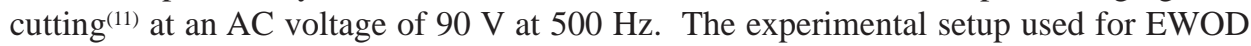
electrode control included the LabVIEW program, a relay box, a signal amplifier, and a CCD microscope for droplet monitoring. AC voltage was generated by a function generator pxi5402 and a relay module pxi2569 from National Instruments ${ }^{\circledR}$ (NI), which were connected to a power amplifier. The movement of the droplet was monitored using the OM system and the CCD microscope set above the EWOD chip.

\subsection{Microheaters in an EWOD system}

As shown schematically in Fig. 1(b), MEMS Pt resistive microheaters were made on the hydrophobic cover of an EWOD chip. To precisely control the temperature of the droplets, two sizes of microheaters were designed to fit the EWOD electrodes, including eight $2 \times 2 \mathrm{~mm}^{2}$ heaters in the middle of the reservoir and two $1.5 \times 1.5 \mathrm{~mm}^{2}$ heaters in the channel. The blue and black pattern in Fig. 2(a) shows the relative positions of the microheaters and EWOD electrodes. Microheaters were fabricated on a glass substrate by e-gun deposition of $\mathrm{Pt}$ for an improved temperature coefficient of resistance (TCR). ${ }^{(24)}$ 


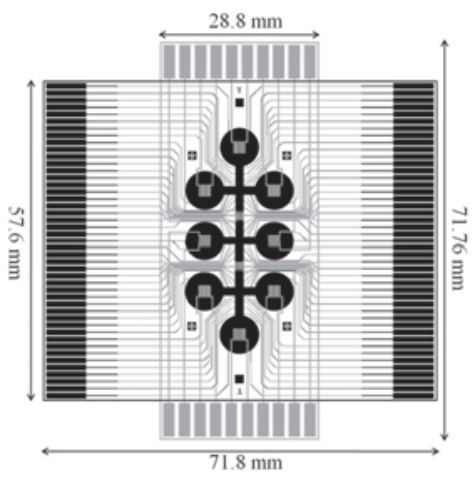

(a)

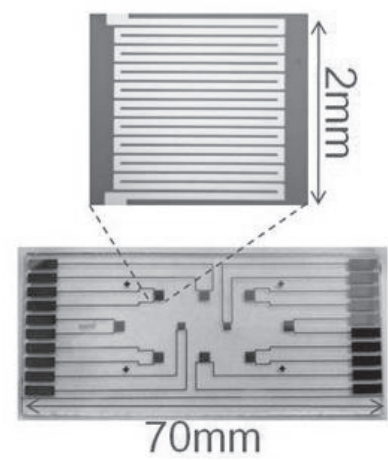

(b)

Fig. 2. Design of the temperature-controllable EWOD microfluidic system. (a) Pattern of the EWOD electrodes and microheaters. (b) Image of microheaters on the cover of an EWOD chip.

After the microheater electrodes were patterned, PECVD $\mathrm{Si}_{3} \mathrm{~N}_{4}$ film (450 nm thickness) was deposited as an insulation layer, and one layer of Teflon (100 nm thickness) was spin coated as a hydrophobic layer. The top views of the microheater device are shown in Fig. 2(b). After the heater circuit design, they were constructed with a bottom EWOD chip to complete the packaging of the temperature-controllable EWOD system. During EWOD droplet manipulation, heaters on the hydrophobic cover were connected to a power supply, and the temperature in different regions of the microfluidic channel was controlled by regulating the input voltage.

\subsection{SNP detection}

The SNP detection has two major parts: DNA probe synthesis on magnetic beads (MBs) and the SNP detection with oligonucleotide ligation. Figure 3 shows a schematic of the DNA probe synthesis on magnetic beads, which was able to identify a single base change from two probes pairing with a DNA template. MBs (Dynabeads M-280, diameter $2.8 \pm 0.2 \mu \mathrm{m}, 12 \%$ iron oxide, Invitrogen) were a commercial product with their surface already modified with oligo $(\mathrm{dT})_{25}$. They were able to hybridize with the primer DNA (5'-CATAATGTTGTAACCAGGCC(A) $)_{10}{ }^{-3}$ '), which was designed to carry oligo (dA) that can bind with oligo $(\mathrm{dT})_{25}$ to become immobilized on the surface of a magnetic bead. The enzyme Klenow Fragment (DNA polymerase II) was used to elongate the oligonucleotide probe on the MBs with dNTP and a reaction buffer, as shown in Fig. 3(a). After $95{ }^{\circ} \mathrm{C}$ treatment for DNA denaturation and washing away the impurities, an MB probe was synthesized as shown in Fig. 3(b).

When the MB probe was synthesized, it could be used in the second part of the SNP detection. The code of the SNP binding site on the MB probe was designed as a " $G$ ", which is able to bind with the target DNA if it contains the SNP code " $C$ ". However, the MB probe was not able to bind with a mismatched DNA, which contains a code " $A$ " on the same detection site. Figure 4(a) shows the SNP detection in a specific gene sequence with Taq DNA ligase. Ligation was successful only when the MB probe perfectly paired with the template. This mechanism was thus usable to examine an SNP polymorphic site on a specific gene sequence, as shown in Fig. 4(b). The MB probe and biotin probe 


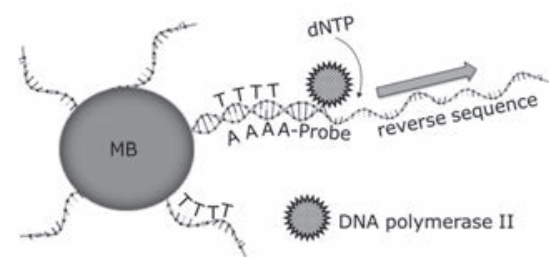

(a)

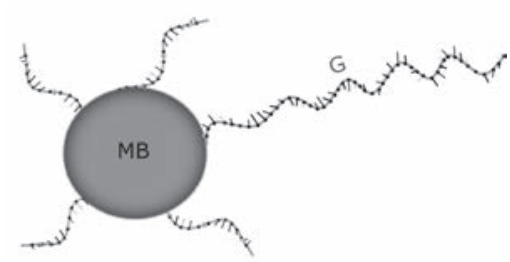

(b)

Fig. 3. MB probe synthesis. (a) Elongation of oligonucleotide probe on MBs by Klenow Fragment. (b) The MB probe was synthesized after washing away the impurities.

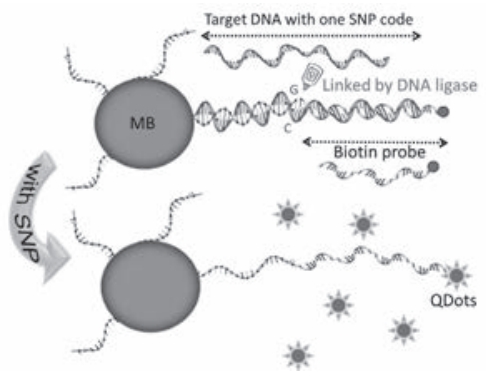

(a)

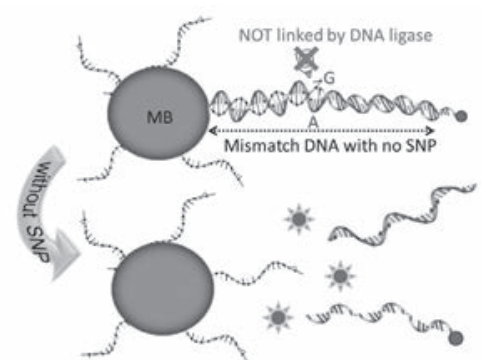

(b)

Fig. 4. Oligonucleotide ligation assay. (a) An MB probe was linked with the biotin probe by DNA ligase because the " $G$ " base on the MB probe is able to hybridize with the " $C$ " base on the target DNA template, and then the MB-biotin probe could be labeled with Qdots. (b) The MB probe could not be linked with a biotin probe by DNA ligase because the " $\mathrm{G}$ " base on the MB probe was not able to hybridize with the "A" base on the mismatched DNA template, and then the MB probe would not be labeled with Qdots.

were both completely paired to the target DNA; the ligase could thus link the MB probe and the biotin probe at $45^{\circ} \mathrm{C}$. After washing away the unlinked impurities, the biotin modified at the biotin probe could covalently attach to streptavidin, which is a biotinbinding protein modified on a fluorescence label (Qdot ${ }^{\circledR} 605$ nanocrystal); we could thus observe a peak emission wavelength of $605 \mathrm{~nm}$ on the MBs. In contrast, the 3' end nucleotide of mismatched DNA was unable to pair with the MB probe; ligation would not occur and the MBs showed no fluorescence signal.

\section{Results and Discussion}

\subsection{Measurement of microheater temperature with an IR scope}

The resistance of a mircoheater is about $20 \mathrm{k} \Omega$. When this EWOD digital microfluidic device with microheaters was connected to the power supply, the droplet temperature on the microheater was measured with an IR scope (QFI InfraScope II) with 6.4 $\times 6.4 \mathrm{~mm}^{2}$ field of view and $25 \mu \mathrm{m}$ pixel resolution; the average temperature of a selected region was calculated automatically using the IR software. When a DI droplet was put on a microheater, we measured the temperature of the droplet with a different input 
voltage and current. Four reservoir microheaters were tested as shown in Fig. 5. The curve diagram shows the relationship between $\Delta T$ of droplets on microheaters and input power. For example, when the input voltage was $30 \mathrm{~V}$, the droplet temperature increased from 30 to $54{ }^{\circ} \mathrm{C}$. The temperature distribution image of a droplet on a microheater was captured using the IR scope, as shown in Fig. 6. After the data was analyzed, the input power to control the temperature of a biochemical reaction could be calculated. In this work, the droplet temperature was stably maintained at $45^{\circ} \mathrm{C}$ for DNA ligation.

\subsection{Manipulation of bioreagent droplets}

The objective of this study was to use a temperature-controllable EWOD digital microfluidic system to detect an SNP in a specific gene sequence. Figure 7 shows the MB probe synthesis by EWOD droplet manipulation. MBs and (dA) ${ }_{10}$-DNA primer droplets were created from EWOD reservoir electrodes, as shown in Figs. 7(a) and 7(b) respectively. Then, these two droplets were merged and transported for primer annealing, as shown in Figs. 7(c) and 7(d). After annealing, a washing protocol with a magnet was required to discard the unlinked impurities. Figure 7(e) shows the MB mixture droplet merging with a washing buffer droplet. Then, a magnet was used for MB extraction, and impurities were separated into a waste droplet by EWOD droplet manipulation, as shown in Fig. 7(f). Finally, the waste droplet was transported into a waste reservoir, as shown in Fig. 7(g). After the washing process, the enzyme Klenow Fragment was used to elongate the oligonucleotide probe on the MBs.

When the MB DNA probe was synthesized, it was used for the SNP detection through oligonucleotide ligation, as shown in Fig. 8. One MB probe droplet was created and transported by EWOD force in Figs. 8(a) and 8(b). Then the target template DNA and biotin probe DNA mixture droplet were created and merged with the MB probe droplet, as shown from Figs. 8(c) to 8(f). A DI water droplet was also created and merged with the reaction mixture. Finally, the mixture droplet was transported into the reaction reservoir containing DNA ligase for DNA ligation, as shown in Figs. 8(h) and 8(i). To ensure that the quantity of magnetic beads sufficed for the ensuing detection of fluorescence, a magnet was used to attract more MBs into a droplet that was then put
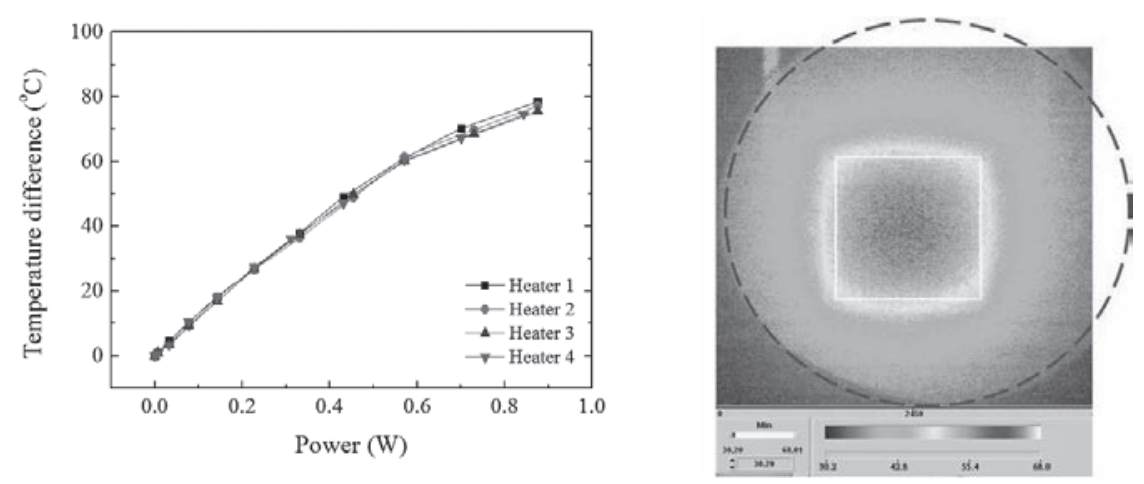

Fig. 5 (left). Relationship between $\Delta T$ of droplets on four microheaters and input power.

Fig. 6 (right). Temperature distribution image of a droplet on a microheater captured using an IR scope. 


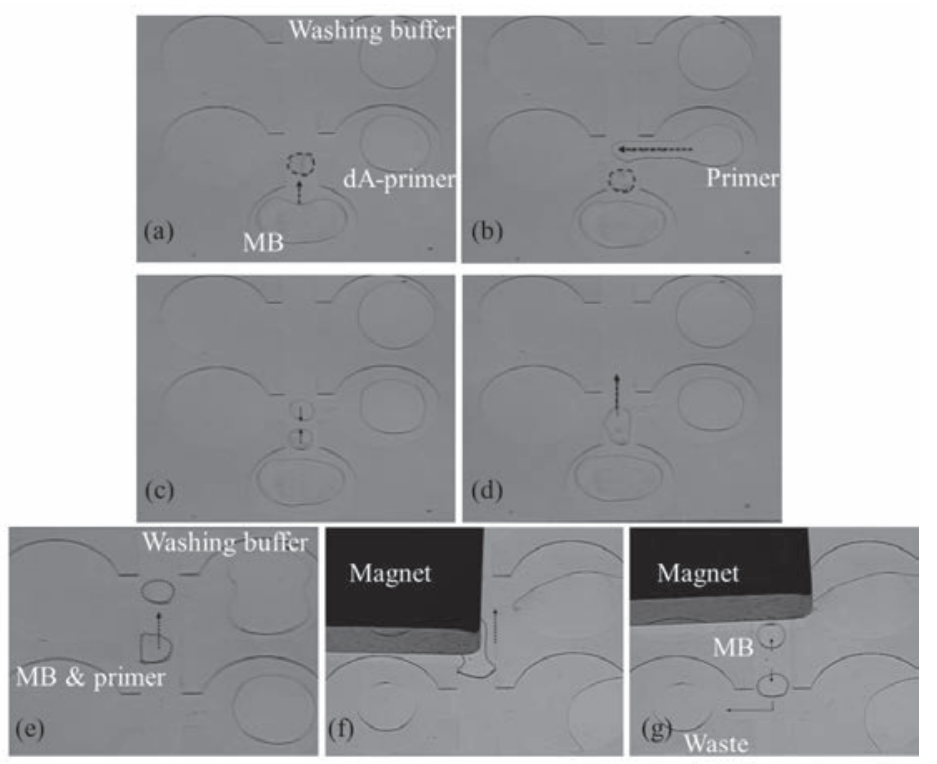

Fig. 7. MB probe synthesis on EWOD chip. (a) MB droplet creation. (b) DNA primer creation. (c) Droplet merging of MBs and DNA primer. (d) Droplet transport. (e)-(g) Repeated MB washing several times with magnet to remove unwanted impurities.

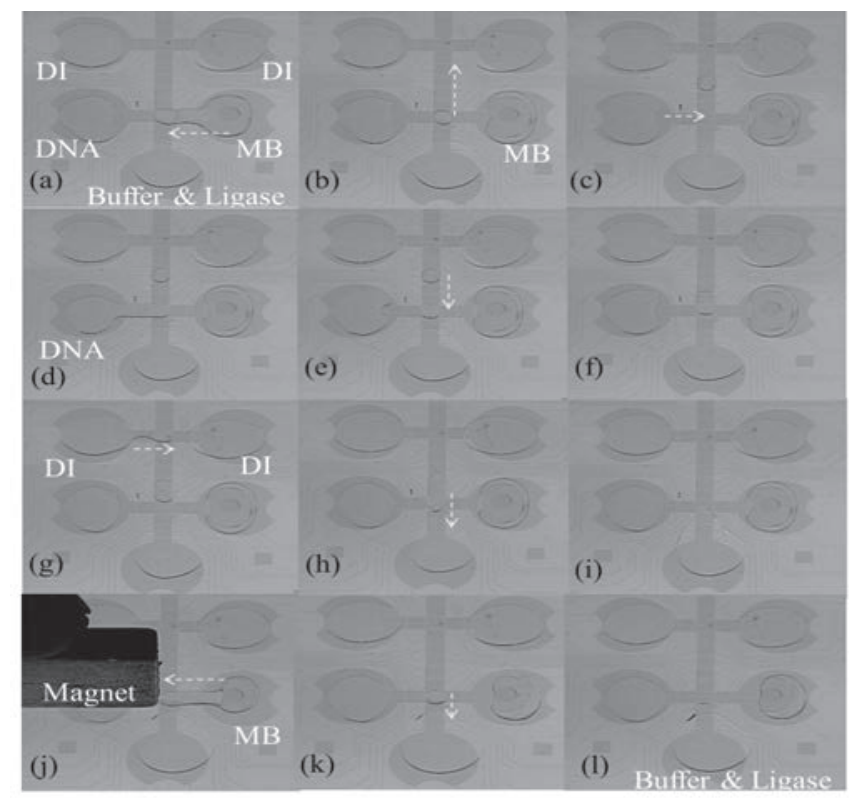

Fig. 8. MB probes were used for the SNP detection by oligonucleotide ligation. (a) and (b) Creation and transport of MB probe droplet. (c) and (d) Creation of target template and biotin probe DNA droplet. (e) and (f) Merging of MB probe and DNA droplets. (g) and (f) Creation of DI water droplet and merging with MBs. (i) Transport of mixture droplet into reaction reservoir containing DNA ligase. (j)-(l) Creation of another MB droplet using magnet to increase the amount of MBs in reaction reservoir. 


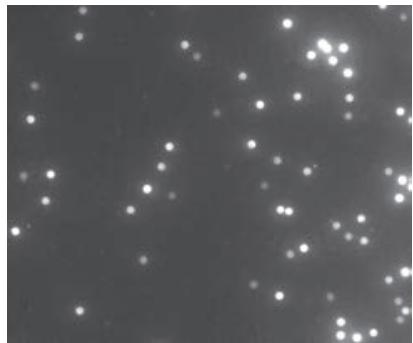

(a)

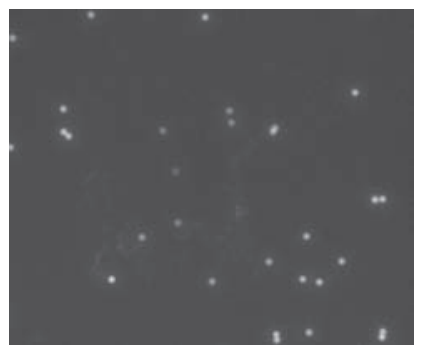

(b)

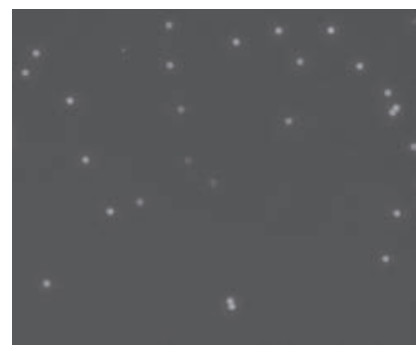

(c)

Fig. 9. Result of MB signal observation under fluorescence microscope. (a) MBs showed a red fluorescence signal because the target DNA used in ligation contained the SNP code "C" in the sequence. (b) MBs did not show a light gray fluorescence signal because the mismatched DNA used in ligation contained no SNP code but "A" in the sequence. (c) No fluorescence signal on MBs when reaction was held at room temperature.

into the reaction reservoir as shown in Figs. 8(j) to 8(l). After EWOD manipulation, the temperature was maintained at $45^{\circ} \mathrm{C}$ using a microheater for DNA ligation.

\subsection{SNP detection}

When the ligation was finished, the MBs were washed and bound with Qdots. If the target DNA contained the SNP code " $C$ " in the sequence, the ligation would be successful, and the biotin probe was able to link with the DNA probe on the MBs. In this way, Qdots could conjugate with biotin and make MBs show a red fluorescence signal, such as in the fluorescence images shown in Fig. 9(a). In contrast, if the mismatched DNA contained no SNP code but "A" in the sequence, ligation failed and there would be no fluorescence signal of the MBs, as shown in Fig. 9(b). To prove that heating with the temperature-controllable EWOD system was a critical step, we show in Fig. 9(c) the fluorescence result of MBs using target DNA containing the SNP code in the ligation but without heating. No fluorescence signal was observed from the MBs; this result proves the importance of the microheater in the SNP detection experiment.

\section{Conclusions}

We developed a temperature-controllable EWOD digital microfluidic system with microheaters designed on the cover of an EWOD chip having coplanar electrodes to provide the appropriate temperature for a biomedical reaction. This digital microfluidic system with a temperature control function is much more convenient to use in an experiment for the SNP detection; the temperature was controlled by adjusting the input power, thus, additional heating devices such as a water bath or an oven were unnecessary. In the future, this temperature-controllable EWOD system is expected to be used in many biomedical diagnoses; this work is thus one approach to the goal of a lab-on-a-chip. 


\section{Acknowledgements}

The authors would like to thank Prof. Fan-Gang Tseng of the Department of Engineering and System Science at National Tsing Hua University, Hsinchu, Taiwan for providing fabrication facilities. This work was partially supported by the National Science Council, Taiwan, under grant no. NSC 100-2628-E-007-014-MY3.

\section{References}

1 K. T. Tang, D. J. Yao, C. M. Yang, H. C. Hao, J. S. Chao, C. H. Li and P. S. Gu: Proc. Olfaction and Electronic Nose 1137 (2009) 86.

2 S. E. Zohora, A. M. Khan and N. Hundewale: Adv. Comp. Inf. Technol. 178 (2013) 177.

3 K. F. Lei: Recent Pat. Nanotechnol. 7 (2013) 81.

4 K. A. Hyun, K. Kwon, H. Han, S. I. Kim and H. I. Jung: Biosens. Bioelectron. 40 (2013) 206.

5 C. H. Chen, D. J. Yao, S. H. Tseng, S. W. Lu, C. C. Chiao and S. R. Yeh: Biosens. Bioelectron. 24 (2009) 1911.

6 C. H. Chen, S. C. Chuang, H. C. Su, W. L. Hsu, T. R. Yew, Y. C. Chang, S. R. Yeh and D. J. Yao: Lab Chip 11 (2011) 1647.

7 R. Sista, Z. S. Hua, P. Thwar, A. Sudarsan, V. Srinivasan, A. Eckhardt, M. Pollack and V. Pamula: Lab Chip 8 (2008) 2091.

8 N. Vergauwe, D. Witters, F. Ceyssens, S. Vermeir, B. Verbruggen, R. Puers and J. Lammertyn: J. Micromech. Microeng. 21 (2011) 054026.

9 S. Park, P. A. L. Wijethunga, H. Moon and B. Han: Lab Chip 11 (2011) 2212.

10 B. Berge: Comptes Rendus de l’Academie des Sciences Serie II. 317 (1993) 157.

11 S. K. Cho, H. J. Moon and C. J. Kim: J. Microelectromech. Syst. 12 (2003) 70.

12 T. H. Lin and D. J. Yao: J. Adhes. Sci. Technol. 26 (2012) 1789.

13 H. C. Lin, Y. J. Liu and D. J. Yao: J. Lab. Autom. 15 (2010) 210.

14 Y. J. Liu, D. J. Yao, H. C. Lin, W. Y. Chang and H. Y. Chang: J. Micromech. Microeng. 18 (2008) 045017.

15 Y. J. Liu, D. J. Yao, H. Y. Chang, C. M. Liu and C. Chen: Biosens. Bioelectron. 24 (2008) 558.

16 G. J. Shah, J. L. Veale, Y. Korin, E. F. Reed, H. A. Gritsch and C. J. Kim: Biomicrofluidics 4 (2010) 044106.

17 P. A. L. Wijethunga, Y. S. Nanayakkara, P. Kunchala, D. W. Armstrong and H. Moon: Anal. Chem. 83 (2011) 1658.

18 W. C. Nelson, I. Peng, G. A. Lee, J. A. Loo, R. L. Garrell and C. J. Kim: Anal. Chem. 82 (2010) 9932.

19 A. H. C. Ng, K. Choi, R. P. Luoma, J. M. Robinson and A. R. Wheeler: Anal. Chem. 84 (2012) 8805.

20 L. Zhu, Y. Y. Feng, X. Y. Ye, J. Y. Feng, Y. B. Wu and Z. Y. Zhou: J. Adhes. Sci. Technol. 26 (2012) 2113.

21 D. Witters, N. Vergauwe, S. Vermeir, F. Ceyssens, S. Liekens, R. Puers and J. Lammertyn: Lab Chip 11 (2011) 2790.

22 C. G. Cooney, C. Y. Chen, M. R. Emerling, A. Nadim and J. D. Sterling: Microfluidi. Nanofluidi. 2 (2006) 435.

23 U. C. Yi and C. J. Kim: J. Micromech. Microeng. 16 (2006) 2053.

24 F. Mailly, A. Giani, R. Bonnot, P. Temple-Boyer, F. Pascal-Delannoy, A. Foucaran and A. Boyer: Sens. Actuators, A 94 (2001) 32. 